\title{
An ancient family of SelB elongation factor-like proteins with a broad but disjunct distribution across archaea
}

\author{
Gemma C Atkinson*, Vasili Hauryliuk, Tanel Tenson
}

\begin{abstract}
Background: SelB is the dedicated elongation factor for delivery of selenocysteinyl-tRNA to the ribosome. In archaea, only a subset of methanogens utilizes selenocysteine and encodes archaeal SelB (aSelB). A SelB-like (aSelBL) homolog has previously been identified in an archaeon that does not encode selenosysteine, and has been proposed to be a pyrrolysyl-tRNA-specific elongation factor (EF-Pyl). However, elongation factor EF-Tu is capable of binding archaeal Pyl-tRNA in bacteria, suggesting the archaeal ortholog EF1A may also be capable of delivering Pyl-tRNA to the ribosome without the need of a specialized factor.

Results: We have phylogenetically characterized the aSelB and aSelBL families in archaea. We find the distribution of aSelBL to be wider than both selenocysteine and pyrrolysine usage. The aSelBLs also lack the carboxy terminal domain usually involved in recognition of the selenocysteine insertion sequence in the target mRNA. While most aSelBL-encoding archaea are methanogenic Euryarchaea, we also find aSelBL representatives in Sulfolobales and Thermoproteales of Crenarchaea, and in the recently identified phylum Thaumarchaea, suggesting that aSelBL evolution has involved horizontal gene transfer and/or parallel loss. Severe disruption of the GTPase domain suggests that some family members may employ a hitherto unknown mechanism of nucleotide hydrolysis, or have lost their GTPase ability altogether. However, patterns of sequence conservation indicate that aSelBL is still capable of binding the ribosome and aminoacyl-tRNA.

Conclusions: Although it is closely related to SelB, aSelBL appears unlikely to either bind selenocysteinyl-tRNA or function as a classical GTP hydrolyzing elongation factor. We propose that following duplication of aSelB, the resultant aSelBL was recruited for binding another aminoacyl-tRNA. In bacteria, aminoacylation with selenocysteine is essential for efficient thermodynamic coupling of SelB binding to tRNA and GTP. Therefore, change in tRNA specificity of aSelBL could have disrupted its GTPase cycle, leading to relaxation of selective pressure on the GTPase domain and explaining its apparent degradation. While the specific role of aSelBL is yet to be experimentally tested, its broad phylogenetic distribution, surpassing that of aSelB, indicates its importance.
\end{abstract}

\section{Background}

Elongation factors EF-Tu in bacteria and EF1A in archaea and eukaryotes bind and deliver aminoacyltRNA (aa-tRNA) to the ribosome. These are the universal components of the EF1 family, which also contains a number of paralogous subfamilies with more restricted taxonomic distributions. EF1 family members all share a common domain structure, of a GTPase (G) domain and two beta barrel domains II and III, which interact

\footnotetext{
*Correspondence: gemma.atkinson@ut.ee

University of Tartu, Institute of Technology, Tartu, Estonia
}

with aa-tRNA. Some EF1 subfamilies also have additional $\mathrm{N}$ terminal or $\mathrm{C}$ terminal domains, responsible for lineage-specific intermolecular interactions. A number of specialized subfamilies recognize and deliver specific tRNAs to the ribosome. Initiator tRNA is specifically recognized by the EF1 family member e/ aIF2 $\gamma$ in eukaryotes and archaea [1]. In nematodes, two mitochondrial EF-Tus are specialized for aa-tRNAs that differ from the canonical cloverleaf tRNA structure comprised of a D arm, $\mathrm{T}$ arm, anticodon arm and accepter loop. Nematode mtEFTu1 specifically binds $\mathrm{T}$ armless tRNAs that are commonly found in metazoan

Ciomed Central

(C) 2011 Atkinson et al; licensee BioMed Central Ltd. This is an Open Access article distributed under the terms of the Creative Commons Attribution License (http://creativecommons.org/licenses/by/2.0), which permits unrestricted use, distribution, and reproduction in any medium, provided the original work is properly cited. 
mitochondria [2], while mtEFTu2 is specialized for D armless Seryl-tRNA, specifically recognizing serine amino acid as well as the tRNA body [3]. Members of the SelB subfamily, close relatives of e/aIF $2 \gamma$ [4] are responsible for binding and delivering selenocysteinyltRNA to the ribosome in selenocysteine-utilizing bacteria, archaea and eukaryotes. At least in the case of bacterial SelB, interaction is specific both to the nature of the tRNA itself and the attached amino acid [5].

The amino acid selenocysteine is structurally similar to cysteine, but contains selenium instead of sulfur, which forms a selenol instead of thiol group. It is chemically more reactive than cysteine [6], and is found in several enzymes (selenoproteins). Selenocysteine has its own tRNA (tRNA ${ }^{\mathrm{Sec}}$ ), but it lacks a dedicated codon in the standard genetic code. Instead, cells employ a specialized mechanism for its integration at a recoded UGA stop codon. Sec-tRNA ${ }^{\text {Sec }}$ forms a complex with the selenocysteine-specific elongation factor SelB and GTP, which in turn binds a structural element in the mRNA (the selenocysteine insertion element, or SECIS). This binding is direct in bacteria, via a $\mathrm{C}$ terminal extension in SelB [7]. In eukaryotic and archaeal SelB (eSelB or EF-Sec), the $C$ terminal extension is shorter and in eukaryotes interacts with the SECIS element via a separate protein, the SECIS binding protein (SBP) [8]. An SBP ortholog is not present in archaea, and a functionally equivalent factor has not been identified, the closest homolog being ribosomal protein S30 [9]. Selenoproteins and the selenocysteine incorporation machinery have a patchy distribution across the tree of life [10]. However, as they are found in eukaryotes, archaea and bacteria, this suggests they were present in the last common ancestor of all life, and were subsequently lost in various lineages [11].

Pyrrolysine is another unusual naturally occurring amino acid [12]. It is a lysine derivative, with a pyrroline ring linked to the lysine side chain. Like selenocysteine, it has a specialized tRNA and is incorporated in response to a termination codon, in this case the 'amber' stop codon UAG [13]. Pyrrolysine has a narrower distribution than selenocysteine, being utilized only in Methanosarcineae archaea and one bacterium, Desulfitobacterium hafniense [13].

In archaea, only some methanogenic archaea are known to utilize selenocysteine, and as such, the synthesis and incorporation machinery (selenophosphate synthetase (SelD), tRNA ${ }^{\text {Sec }}$ (SelC) and SelB) are only found in these organisms [10]. A paralog of archaeal SelB (aSelB) has been identified in Methanosarcinales, and proposed to be a hitherto unidentified pyrrolysine-specific elongation factor (EF-Pyl) [14]. A structure of the putative EF-Pyl was determined and deposited in the protein data bank in 2007 (PDB ID 2ELF[15]). However, bacterial EF-Tu has been found to be capable of binding archaeal Pyl-tRNA, suggesting that the archaeal ortholog of EF-Tu, aEF1A, may also be capable of delivering Pyl-tRNA to the ribosome without the need of a specialized factor [16].

We have identified EF-Pyl/aSelB-like homologs (herein referred to as aSelBL) in a range of archaea that is broader than the distribution of selenocysteine and pyrrolysine. We have carried out comparative phylogenetic and sequence analysis of the aSelBL subfamily. Strong structural similarity with aSelB and conservation of tRNA and ribosomal RNA interaction sites suggests that in some archaea, aSelBL may be a functional translation elongation factor. However, disrupted $\mathrm{G}$ domains in subgroups of aSelBL raise the possibility that the aSelB proteins are not typical molecular switches like classical translational GTPase elongation factors. Their taxonomic distribution, while non-continuous, is broad, and generally (although not exclusively) associated with methanogenic high-cysteine archaea. We discuss possible roles for these unusual proteins.

\section{Results}

\section{Phylogenetic analysis}

Sequence searching revealed the presence of homologs of archaeal SelB in the genomes of many Euryarchaea, the recently designated phylum Thaumarchaeota [17], and Sulfolobales and Thermoproteales within Crenarcheatoa (Figure 1, Table 1, Additional files 1 and 2). Thus, aSelB homologs are present, although with a disjunct distribution, in all three major lineages of archaea [17]. ML and BI phylogenetic analysis of bacterial SelB, aSelB, EF-Tu and aIF $2 \gamma$ shows that the aSelB homologs form a monophyletic group with full support (BIPP 1.0, MLBP 100\%, Additional file 1). 'True' aSelB (i.e. the previously identified and characterized aSelB selenocysteine elongation factor) from Methanopyrus kandleri represents a sister group to a clade that comprises 'true' aSelB from Methanococcales, plus a divergent clade henceforth referred to as aSelB-like (aSelBL). The monophyly of Methanococcale aSelB + aSelBL to the exclusion of $M$. kandleri aSelB is not strongly supported (0.88 BIPP, 68 MLBP). However, aSelBL is fully supported as monophyletic and is also supported by a 15 amino acid deletion in the GTPase domain, relative to "true" aSelB (alignment positions 3457, Figure 2). Species of Methanococcus and Methanocaldococcus have both aSelB and aSelBL. Thus it appears that aSelBLs are not orthologs of aSelB, but are paralogs or xenologs, originating via gene duplication or lateral transfer, rather than speciation. Only M. kandleri has aSelB without a copy of aSelBL.

Phylogenetic analysis of aSelB/aSelBL alone was carried out to reveal the internal branching structure of the subfamily, using more universally alignable homologous amino acid positions (Figure 1). Candidatus 


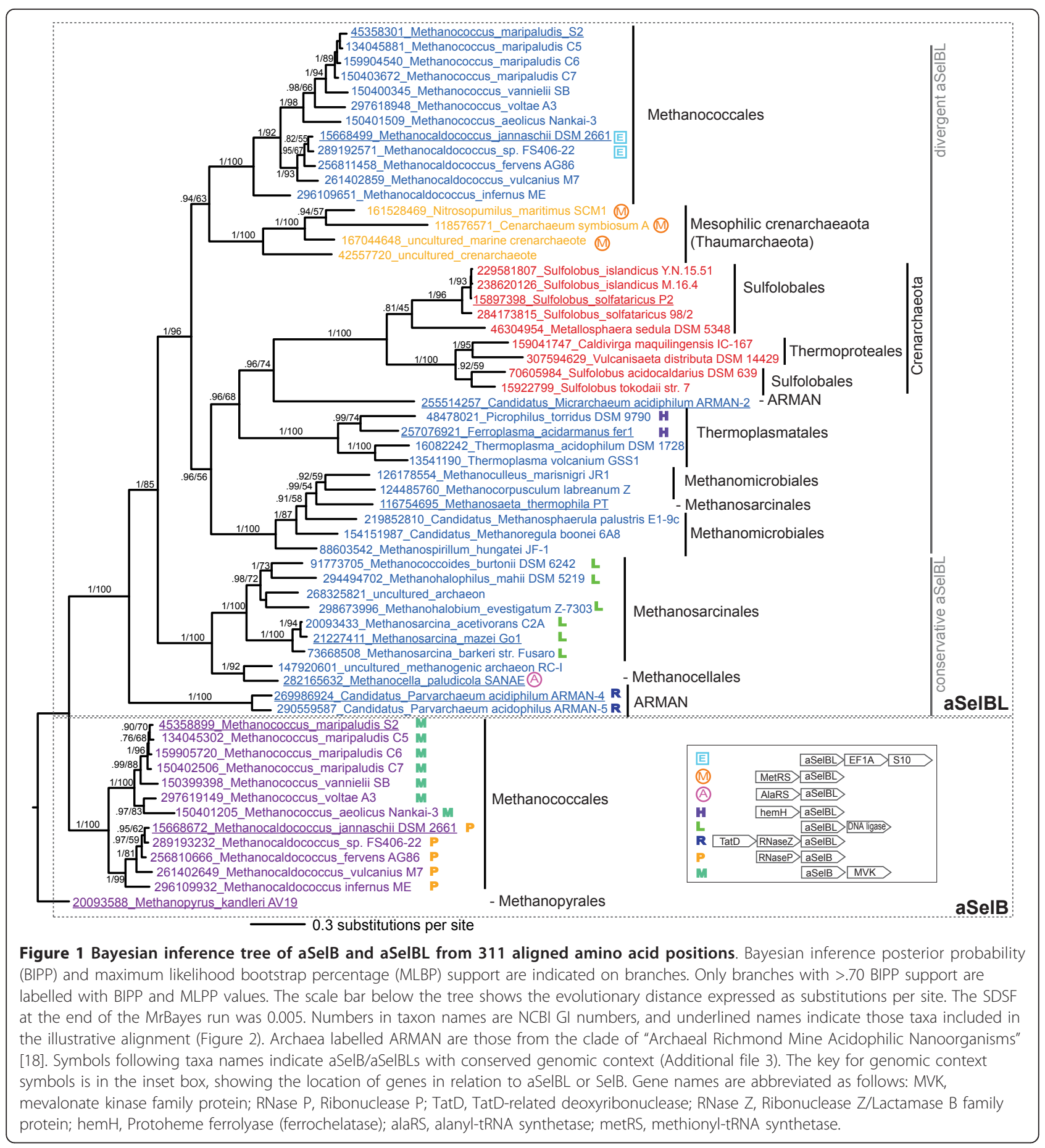

Parvarchaeum acidiphilum ARMAN-4 and -5 form a sister group to the remaining aSelBLs, which have strong BI and moderate ML support for monophyly (1.0 BIPP, 85\% MLBP, Figure 1). The ARMAN (Archaeal Richmond Mine Acidophilic Nanoorganisms) bacteria are proposed to branch near the root of the Euryarchaeota [18]. This suggests that aSelBL may have originated within or before the lineage leading to Euryarchaeaota. However, one of the
ARMAN bacteria, Candidatus Microarchaeum acidiphilum ARMAN-2 is nested deeply within aSelBL, grouping with the Crenarcheaota clade with moderate support (0.96 BIPP, 74\% MLBP, Figure 1). Both the Thaumarchaeota and Crenarchaeota $+M$. acidiphilum clades are nested strongly within Euryarchaeota (1.0 BIPP, 96\% MLBP, Figure 1). This does not agree with currently accepted deep archaeal phylogeny $[17,19]$. Thus, the aSelBLs of 
Table 1 Distribution of aSelBL, aSelB, SeID and pylS across archaeal full genomes

\begin{tabular}{|c|c|c|c|c|c|}
\hline \multicolumn{2}{|c|}{ Taxonomic groupings* } & \multicolumn{4}{|c|}{ Proteins identified* } \\
\hline Thaumarchaeota & Nitrosopumilus_maritimus SCM1 & aSelBL & & & \\
\hline \multirow[t]{13}{*}{ Crenarchaeota } & Desulfurococcales & - & & & \\
\hline & $\underline{\text { Sulfolobales }}$ & & & & \\
\hline & Metallosphaera sedula DSM 5348 & aSelBL & & & \\
\hline & Sulfolobus acidocaldarius DSM 639 & aSelBL & & & \\
\hline & Sulfolobus islandicus & aSelBL & & & \\
\hline & Sulfolobus solfataricus & aSelBL & & & \\
\hline & Sulfolobus tokodaii str. 7 & aSelBL & & & \\
\hline & Thermoproteales & & & & \\
\hline & Caldivirga maquilingensis IC-167 & aSelBL & & & \\
\hline & Pyrobaculum (multiple species) & & & & \\
\hline & Thermofilum pendens Hrk 5 & & & & \\
\hline & Thermoproteus neutrophilus V24Sta & & & & \\
\hline & Vulcanisaeta distributa DSM 14429 & aSelBL & & & \\
\hline \multirow[t]{35}{*}{ Euryarchaeota } & Archaeoglobales & - & & & \\
\hline & Halobacteriales & - & & {$[S e l D]^{* *}$} & \\
\hline & Methanobacteriales & - & & & \\
\hline & Methanococcales & & & & \\
\hline & Methanocaldococcus fervens AG86 & aSelBL & aSelB & SelD & \\
\hline & Methanocaldococcus infernus ME & aSelBL & aSelB & SelD & \\
\hline & Methanocaldococcus jannaschii DSM 2661 & aSelBL & aSelB & SelD & \\
\hline & Methanocaldococcus sp. FS406-22 & aSelBL & aSelB & SelD & \\
\hline & Methanocaldococcus vulcanius M7 & aSelBL & aSelB & SelD & \\
\hline & Methanococcus aeolicus Nankai-3 & aSelBL & aSelB & SelD & \\
\hline & Methanococcus maripaludis & aSelBL & aSelB & SelD & \\
\hline & Methanococcus vannielii SB & aSelBL & aSelB & SelD & \\
\hline & Methanococcus voltae $A 3$ & aSelBL & aSelB & SelD & \\
\hline & Methanomicrobiales & & & & \\
\hline & Candidatus Methanoregula boonei $6 A 8$ & aSelBL & & & \\
\hline & Methanocorpusculum labreanum Z & aSelBL & & & \\
\hline & Methanoculleus marisnigri JR1 & aSelBL & & & \\
\hline & Methanosphaerula palustris E1-9c & aSelBL & & & \\
\hline & Methanospirillum hungatei JF-1 & aSelBL & & & \\
\hline & Methanopyrales & & & & \\
\hline & Methanopyrus kandleri AV19 & & aSelB & SelD & \\
\hline & Methanosarcinales & & & & \\
\hline & Methanococcoides burtonii DSM 6242 & aSelBL & & & pyls \\
\hline & Methanohalobium evestigatum Z-7303 & aSelBL & & & pyls \\
\hline & Methanohalophilus mahii DSM 5219 & aSelBL & & & pyls \\
\hline & Methanosaeta thermophila PT & aSelBL & & & {$[p y \mid S]^{* *}$} \\
\hline & Methanosarcina acetivorans $C 2 A$ & aSelBL & & & pyls \\
\hline & Methanosarcina barkeri str. Fusaro & aSelBL & & & pyls \\
\hline & Methanosarcina mazei Go1 & aSelBL & & & pyls \\
\hline & Thermococcales & - & & & \\
\hline & Thermoplasmales & & & & \\
\hline & Ferroplasma acidarmanus fer 1 & aSelBL & & & \\
\hline & Picrophilus torridus DSM 9790 & aSelBL & & & \\
\hline & Thermoplasma acidophilum DSM 1728 & aSelBL & & & \\
\hline & Thermoplasma volcanium GSS1 & aSelBL & & & \\
\hline
\end{tabular}

Nanoarchaeota Nanoarchaeum equitans Kin4-M

*Orders that carry either aSelB or aSelBL are expanded to show species and strain (full data in Additional file 2).

**Square brackets indicate divergent or partial sequences (details in text). 


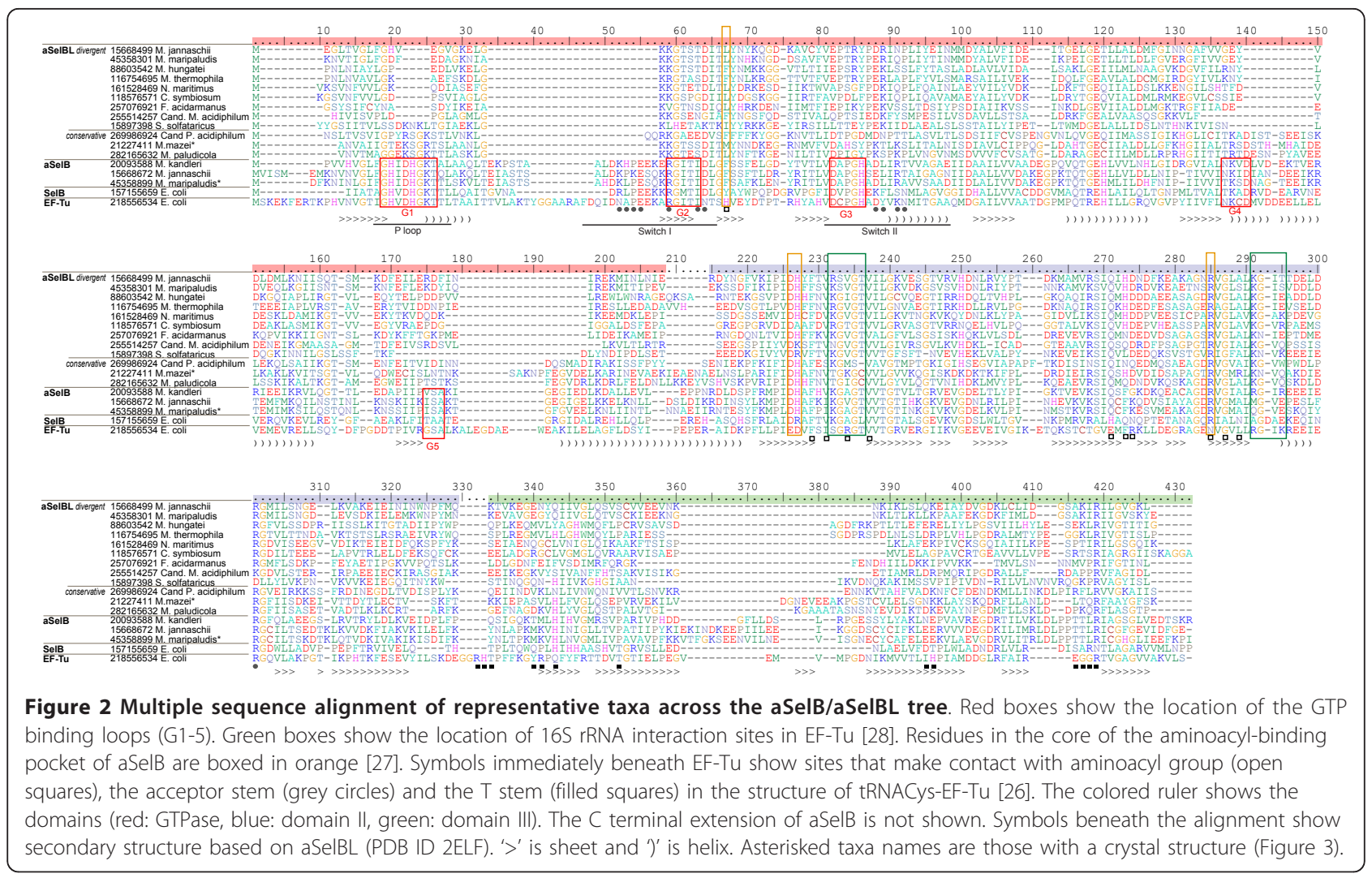

Thaumarchaeota, Crenarchaeota and $M$. acidiphilum appear to have arisen as a result of duplication and loss, or horizontal gene transfer (HGT) from Euryarchaea. Another possible case of HGT may have occurred in the lineage to Methanosaeta thermophila, which branches within the Methanomicrobiales with full BIPP and MLBP support, instead of its home clade of Methanosarcinales $[17,19]$, which is otherwise fully supported as a monophyletic group (Figure 1).

BLASTP searches with pyrrolysine synthetase (PylS) confirmed that pyrrolysine synthesis is limited to the Methanosarcinales (Table 1). In one Methanosarcinale, Mathenosaeta thermophila, only a partial PylS sequence was identified from a short sequence read, as previously noted [20]. The presence of selenophosphate synthetase (SelD) in a genome is a signature for selenium utilization [10]. BLASTP searches with SelD confirmed that selenium utilization is limited to Methanococcales and Methanopyrus (Table 1). However one divergent SelDlike homolog was also found in Haloarcula marismortui, as previously identified [21].

\section{Sequence and structure of aSelBL}

The most striking feature of the aSelBL subfamily alignment is a GTPase domain that deviates from the classical architecture, characterized by the presence of
GTP-binding motifs (G1-G5) [22]. In particular, G1 (within the phosphate binding (P) loop), which binds the $\alpha$ - and $\beta$-phosphates of both GDP and GTP has experienced a 4-5 amino acid deletion in some aSelBLs relative to other EF1 family members (alignment positions 21-25, Figure 2).

The extent of the $\mathrm{G}$ domain disruption follows the phylogeny, with distinct clades in the tree having specific sequence patterns. The most conservative aSelBLs are the clades of Candidatus Parvarchaeum acidiphilum ARMAN-4 and -5, and Methanocellales + Methanosarcinales ("conservative aSelBL", Figure 1). These taxa retain some conserved residues of the G1 and G4 motifs, although with less overall sequence conservation in the motifs than SelB and aSelB (Figure 2). The monophyly of the more divergent aSelBLs ("divergent aSelBL", Figure 1) is well supported in the tree (1.0 BIPP, 96\% MLBP, Figure 1), and independently supported by a 10-14 amino acid indel (alignment positions 140-149, Figure 2). This region is conserved in conservative aSelBL, aSelB and other EF1 family members, but deleted in divergent aSelBLs (Figures 1, 2). Similarly, the G1 (P loop) region contains a large deletion in the divergent aSelBLs (alignment positions 20-25, Figure 2), with the exception of the Crenarchaeota. The latter do not have the deletion, however sequence homology 
between Crenarchaeota and the other aSelBLs is ambiguous in this region, and it is possible that the lack of the deletion may actually correspond to a secondary insertion.

Despite the large deletions, regions within the $\mathrm{G}$ domain, specifically sequence motifs G2 and G3, are conserved in aSelBL, however often with different residues relative to aSelB (Figure 2). In P-loop GTPases, the threonine of the G2 "RGITI" motif (within the switch I or the effector loop) and the glycine of G3 "DXPGH" motif (within switch II) bind the $\gamma$-phosphate of GTP, thus discriminating the nature of the bound nucleotide. Switches I and II are at the core of GTPase function, undergoing conformational changes between GTP and GDP binding states [23]. The "RGITI" G2 motif common to trGTPases is conserved at the $60 \%$ level in aSelBL as "KGTXX" (where X is any unconserved amino acid). Similarly, "DXPGH" of G3 has a $60 \%$ consensus of "E/DPXXX" in aSelBL. The histidine of G3 (position 86, Figure 2) is unconserved in aSelBL, although it is critical for GTP hydrolysis, and therefore universally conserved in other translational GTPases [24]. In EF-Tu, it is the positioning of His84 between Val20 of the P loop and Ile60 of Switch I by the sarcin/ricin loop (SRL) of the ribosome that activates the GTPase [24]. The differential conservation between aSelBL and other translational GTPases suggests that aSelBL has diverged in GTPase function, both in terms of GTP/GDP discrimination and GTP hydrolysis, but that the divergence is not due to a general degradation of the GTPase domain. The switch I and II regions are also involved in binding the tRNA acceptor stem, providing a physical link between tRNA and GTP binding to EF-Tu (Figure 2, [25,26]). Thus the functional divergence in aSelBL in switches I and II may have involved modification or refinement of tRNAbinding capabilities.

One of the most conservative aSelBLs, M. mazei aSelBL has been subjected to X-ray analysis. Diffraction data were collected in 2004, and the aSelBL structure was determined and deposited in the protein databank in 2007 (PDB ID 2ELF) [15]. Structural alignment with aSelB [27] shows that the overall structures are remarkably similar (Figure 3). Indeed, even in the GTPase domain, the structures are completely superimposable, with the exception of the switch I region, which is disordered in aSelB [27]. The aSelBLs do not, however, carry the $C$ terminal extension that is present in all other SelBs and is required for SECIS or SECIS-binding protein recognition (Figure 3). Thus, aSelBLs are unlikely to be able to respond to SECIS, or similar elements in the mRNA.

Domains II and III are much more conserved than the G domain across aSelB/aSelBL (Figure 2). Structural and mutational anlaysis shows that in M. maripaludis aSelB, the core of the aminoacyl-binding pocket is formed by

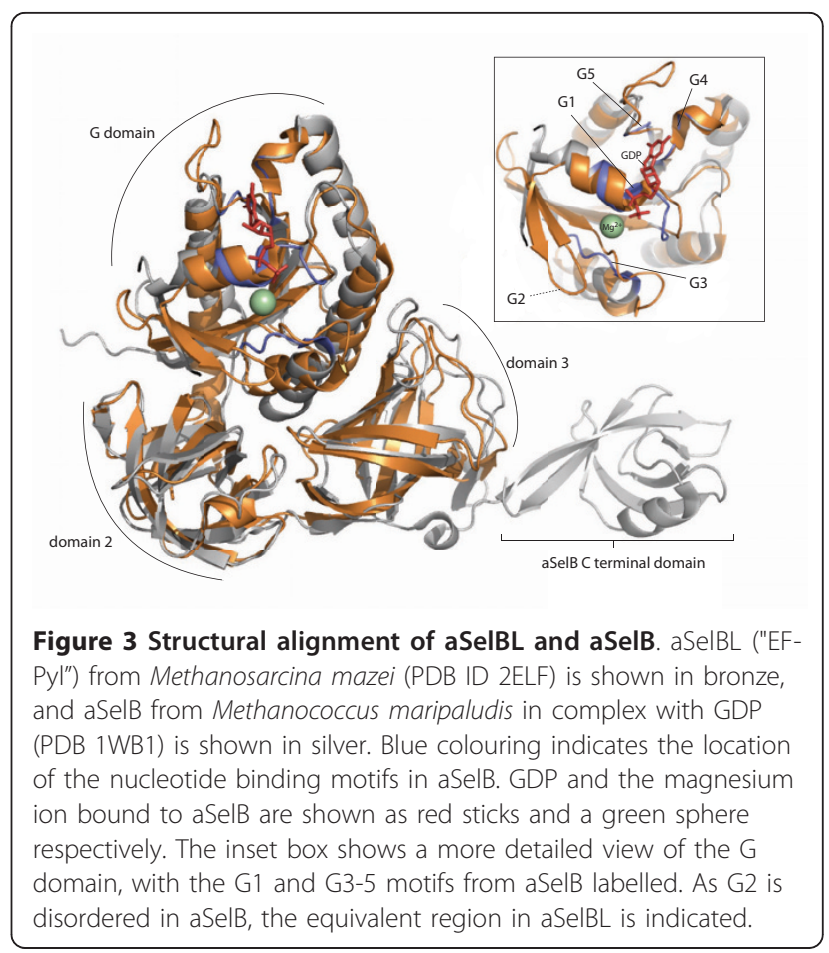

residues Asp191, His192 and Arg247 [27] (orange boxes, Figure 2). In aSelBL, Asp191 and His192 (positions 226-227, Figure 2) are universally conserved, with the exception of the longest branched aSelBLs in the tree (Sulfolobales + M. acidiphilum), which lack His192. Arg247 (position 285, Figure 2) is universally conserved. Similarly, sites that make contact with aminoacyl group, the acceptor stem and (to a lesser extent) the $\mathrm{T}$ stem in the structure of tRNACys-EF-Tu [26] are often well conserved between aSelB and aSelBL (Figure 2). The structure of aSelBL can be superposed on that of the EF-Tu-Cys-tRNACys ternary complex [26], showing that the aminoacyl binding pocket is also structurally well conserved (Figure 4). Thus, aminoacyl-tRNA binding capabilities appear to be retained in aSelBL.

A particularly interesting feature of the aSelB structure is Phe51 (position 67, Figure 2), which protrudes into the aminoacyl binding pocket [27]. This phenylalanine, which can be replaced by tyrosine in some SelBs, is proposed to form a hydrophobic "lid" that protects selenocysteine from oxidation [27]. Such a lid may also be a feature of aSelBLs, which all carry a Phe or Tyr at position 67 and/or position 68 (Figure 2). Although Tyr33 (at position 68 in Figure 2) is facing away from the aminoacyl-binding pocket in the aSelBL structure (Figure 4), this residue is proposed to be flexible in aSelB [27] and its orientation could change upon aa-tRNA-binding.

The structure of aa-tRNA-EF-Tu on the ribosome reveals specific interactions between the elongation 


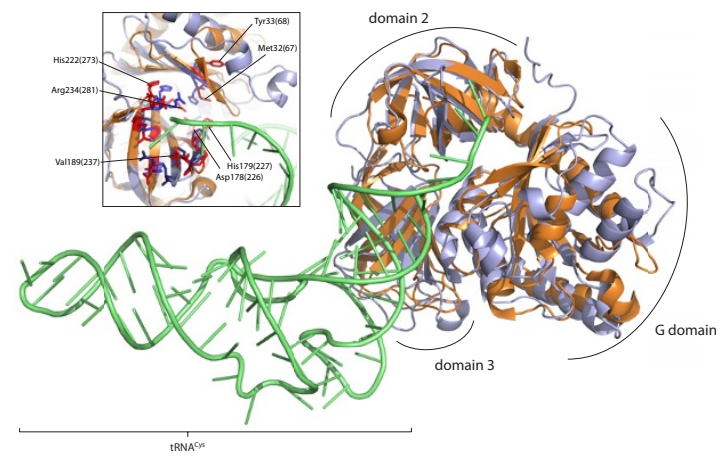

Figure 4 Structural alignment of aSelBL and EF-Tu-CystRNACys. aSelBL ("EF-Pyl", PDB ID 2ELF) is shown in bronze and EFTu-Cys-tRNACys (PDB 1B23) is shown in pale blue and green, for EFTu and tRNA respectively. The inset box shows a more detailed view of the aminoacyl-binding pocket. Residues lining the pocket are shown in red for $\mathrm{aSelBL}$, with equivalent EF-Tu sites in blue. Residue labels use M. mazei numbering, with Figure 2 alignment numbers in parentheses.

factor and ribosomal RNA [28]. Two patches in particular in domain II of EF-Tu associate with the shoulder domain of $16 \mathrm{~S}$ rRNA [28]. Both of these patches are well conserved in aSelBL (green boxes, Figure 2), suggesting ribosome binding may also have been retained in aSelBL evolution.

\section{Genomic context}

The genomic context of a gene of interest can provide clues about function, as functionally related genes are often co-located and co-transcribed in archaea as well as bacteria [29]. Pyrrolysine biosynthesis and utilisation genes cluster together [30], as do ribosomal RNA genes [31] and elongation factors in some archaea [32]. Genes flanking the $a S e l B L$ gene are mostly unconserved in context (Additional file 3). However there are some co-localizing genes that are transcribed in the same direction as aSelBL with no intervening gene on the complementary strand and are conserved in multiple species (Figure 1 Additional file 3). These encode genes with various functions, and in general, the significance (if any) of their colocalization with $a S e l B L$ is uncertain. Methanosarcinales carry an ATP-dependent DNA ligase upstream of a SelB, often following a CBS (Cystathionine Beta Synthase) domain-containing protein. The aSelBs of Picrophilus torridus and Ferroplasma acidarmanus are immediately downstream of ferrochelatase hemH, and downstream of two ribonucleases in Candidatus Parvarchaeum acidiphilum ARMAN-4 and -5 (Figure 1, Additional file 3). Other conserved flanking genes are translation-associated. Three of the four Thaumarchaea (Nitrosopumilus maritimus, Cenarchaeum symbiosum and uncultured marine crenarchaeote HF4000 APKG7F19) encode a methionyl-tRNA synthetase upstream of aSelB, and Methanocella paludicola carries an upstream alanyltRNA synthetase. Additionally, Methanocaldococus Jannaschii and Methanocaldococcus sp. FS406-22 aSelBs are upstream of the ribosomal protein S10 and elongation factor $1 \mathrm{~A}$.

\section{Discussion}

\section{Origin of aSelBL}

Given the wide distribution of aSelBL in methanogens, and its presence in all currently known species of Thaumarchaeaota, the original duplication of the ancestral aSelB appears to be very ancient, occurring very early in archaeal evolution, possibly before the archaeal last common ancestor (aLCA). However, the well supported nesting of Thaumarchaeote aSelBL within methanogens appears to suggest HGT. This is a surprising association, as it would require that the transfer occurred before the divergence of all currently sampled genera within the Thaumarchaeaota phylum. The presence of aSelBL in only Sulfolobales and two Thermoproteales of Crenarchaeota may be due to one or more horizontal transfers into Crenarchaeota. Alternatively, as there are only branches with weak to moderate support (0.94-0.96 BIPP, 56-74\% MLBP) separating Crenarchaeota and Thaumarcaheota, aSelBL may have been inherited vertically from the ancestor of Crenarchaeota and Thaumarcaheota. In this case, loss of aSelBL must have occurred in the Desulforococcale lineage, and at least once more to account for its absense in Pyrobaculum, Thermofilum and Thermoproteus genera. Within Euryarchaeota, aSelBL was also lost or was never present in Halobacteriales, Archaeoglobus, Methanobacteriales, Methanopyrus (Table 1, Additional file 2). At present, it is impossible to retrace the origin of aSelBL in all archaea, but it is likely that both differential loss and HGT have been involved.

Assuming SelB was present in the LCA of archaea as previously suggested [11], classical aSelB must also have been lost in multiple lineages, as it is only found in Methanococcales and Methanopyrus. As aSelB has experienced more loss than aSelBL, the function of the latter seems more critical than the former in a number of archaea. Indeed, aSelBL is found in some of the smallest genomes of archaea, for example Picrophilus torridus (1.5 megabases) and Methanocaldococcus infernus (1.3 megabases), and also the largest (M. acetovorans, 5.8 megabases). This suggests that loss of aSelBL has not been due to general genome size reduction.

\section{Possible functions of aSelBL}

Genomic context that includes genes for aminoacyltRNA synthetases, ribosomal protein S10 and EF1A suggests that at least in some cases, aSelBL may still be 
involved in some aspect of translation. Indeed the structure of conservative aSelBL is strikingly similar to aSelB and patterns of sequence conservation suggest that all aSelBLs are capable of binding the ribosome and aminoacyl-tRNAs. However, the major disruptions in the GTPase domain raise the question of whether aSelBL can function as a classical GTPase. Additional differences in the $\mathrm{G}$ domains within the aSelBL group also suggest that its function has diverged to some extent between conservative and divergent aSelBLs.

Although the aSelBLs arose from a selenocysteinespecific elongation factor, they are unlikely to have retained this role. Firstly, there is no $\mathrm{C}$ terminal extension with which to recognize the SECIS or SECIS-binding proteins. Secondly and more importantly, other components of the incorporation machinery are not present in most of the aSelBL-containing taxa (Table 1, [10]), and neither are selenoproteins [33]. aSelBL has previously been proposed to be a pyrrolysine-specific elongation factor in Methanosarcinales [14]. However, in archaea, only Methanosarcinales utilise pyrrolysine and encode the pyrrolysyl-tRNA synthetase gene ( $p y l S$, Table 1). Thus, as with the selenocysteine usage distribution, the pyrrolysine distribution is only a subset of the full aSelBL distribution.

Despite the weight of evidence suggesting that aSelBL is not a selenocysteine-tRNA elongation factor, the amino acids responsible for selenocystine specificity are well conserved in aSelBL. This suggests that aSelBL is still specific for a single amino acid, with the most likely candidate being cysteine, given its structural similarity to selenocysteine. The conserved, positively charged residues that line the aminoacyl binding pocket of aSelBL could potentially accommodate the negative thiol group of cysteine, just as they accommodate the negative selenol group in classical SelBs. Indeed, bacterial SelB cannot entirely distinguish sulfur from selenium, and is able to bind Cys-tRNASec tightly enough to allow for Cys-tRNASec delivery to the ribosome [34]. This raises the possibility that after duplication of the ancestral aSelB/aSelBL protein, aSelBL was recruited for a process requiring Cys-tRNA recognition, losing its SECIS or SBP-binding domain in the process. Following loss of selenium utilization, aSelB was lost from many archaea, but aSelBL was maintained in many lineages, and horizontally transferred to others.

This raises the question of what cysteine-associated mechanisms(s) aSelBL may be involved with. Cysteine is overrepresented in methanogenic archaea, which contain many iron sulfur cluster proteins, coordinating iron via cysteine-rich motifs [35]. Some methanogenic archaea lack the canonical class I cysteine-tRNA synthetase (CysRS). In these archaea, Cysteine is synthesized via a recently discovered tRNA-dependent pathway. O-phosphoseryl-tRNA synthetase (SepRS) ligates O-phosphoserine to tRNACys, which is converted to Cys-tRNACys by Sep-tRNA:CystRNA synthase (SepCysC) [36,37]. It has been suggested that the tRNA-dependent cysteine biosynthesis pathway is a strategy to protect cysteine from thermal degradation, by limiting the amount of free cysteine [38]. Indeed, there is a correlation between the presence of SepRS and high cysteine content proteomes [39]. Potentially any cysteinyltRNA binding protein may aid in this protective function. Therefore the role of aSelBL may be primarily to bind and protect the highly labile cysteine from degredation. Consistent with this, the protective hydrophobic "lid" of the aminoacyl-binding pocket of aSelB that could protect selenocysteine/cysteine from oxidation also appears to be present in aSelBL. There is an association of aSelBL with high cysteine methanogens (Additional files 2 and 4). However, this clustering may simply be due to the common ancestry of methanogens, as the non-methanogens (Thermoplasmatales, Sulfolobales and Thaumarchaeota) that also encode aSelBL do not show a similar enrichment in cysteine (Additional file 4).

With its conservation of ribosome, tRNA and aminoacyl-binding sites, aSelBL may be a specialized elongation factor for incorporation of cysteine or another amino acid. Classical EF1 family elongation and initiation factors bind to the ribosome in complex with aa-tRNA and GTP, with complex formation between the GTPase and aa-tRNA strongly promoted in the presence of GTP [40-42]. In accordance with this paradigm, formation of the bacterial SelB*Sec-tRNA ${ }^{\text {Sec*}_{*}}$ GTP ternary complex is strongly promoted by GTP [5]. However, when tRNA ${ }^{\text {Sec }}$ is charged with an amino acid other than selenocysteine, i.e. serine, which acts as a precursor in selenocysteine biosynthesis [43], the interaction becomes insensitive to G nucleotides, effectively decoupling the GTPase function from the tRNA binding function [5].

This behaviour of bacterial SelB invites us to speculate on possible scenarios for the functional evolution of aSelBL. Following duplication of aSelB, the redundant aSelBL may have acquired a new aa-tRNA partner. This would have led to loss of the GTP-dependence of ternary complex formation, due to the absolute selenocysteine specificity of the parental SelB [5]. Loss of the functionality of the GTPase cycle would have led to a relaxation of selective pressure on the $G$ domain in terms of GTP/GDP discrimination, but continued selection for aa-tRNA-binding capabilities, resulting in an aSelBL that retains the classical GTPase fold (Figure 3). The conserved motifs G1-5 of the G domain are poorly conserved in aSelBL, including the histidine of switch II that is critical for GTP hydrolysis [24]. Thus aSelBL appears unlikely to hydrolyse GTP on the ribosome via the same mechanism as other proteins of the translational GTPase superfamily. However, the functionality 
of the aSelBL G domain needs to be addressed experimentally, as these proteins may still hydrolyse GTP or another nucleotide via a hitherto unknown mechanism. Alternatively, they may be non-enzymatically delivering aa-tRNA to the ribosome. This would not be without precedent, as a eukaryotic factor has recently been discovered that delivers aa-tRNA to the ribosome independently of GTP [44]. We also can not exclude the possibility that aSelBL may be involved in an aspect of tRNA biochemistry not directly related to protein synthesis. There is a wide variety of tRNA processing mechanisms in archaea [45]. For example, tRNA splicing, reassembly of split tRNA genes, the addition of a G-1 residue in $\mathrm{TRNA}^{\text {His }}$ and adding the 3' CCA end, the three bases required for aminoacylation.

\section{Conclusions}

Although the function of aSelBL remains obscure, the wide distribution of these proteins among diverse archaea suggests that they have functions of some significance. This important enzyme has so far been overlooked experimentally, but future characterization may reveal important mechanisms of translational control in archaea.

\section{Methods}

Sequences were retrieved by BLASTP searches against archaeal genomes in NCBI genomes (Additional file 2). Methanosarcina Mazei ("EF-Pyl"), Methanospirillum hungatei and Vulcanisaeta distributa aSelBLs were used as queries, with an E value cut-off of 1e-8. Additional BLASTP searches were carried out against the NCBI nr database to retrieve any additional archaeal SelB (aSelB) -like sequences from incomplete genome projects. This also confirmed that aSelB-like sequences are only found within archaea. The identity of homologs as close relatives of SelB, rather than other paralogs within the translational GTPase (trGTPase) superfamily was verified by scanning against the translational GTPase database [46]. The resulting "aSelB" data set contained 60 sequences from 47 archaea. To root the aSelB phylogeny, 30 additional sequences from bacterial SelB, elongation factor $\mathrm{Tu}(\mathrm{EF}-\mathrm{Tu})$ and archaeal initiation factor $2 \gamma$ (aIF2 $\gamma$ ) were retrieved from the translational GTPase database [46] and added to the data set.

Sequences were aligned using MAFFT v6.234b with strategy L-INS-I [47] for use in phylogenetic analyses. Poorly aligned and gap-rich regions of the alignment were identified by eye and excluded from the analyses. The 60 sequence $\mathrm{aSelB}$ and 90 sequence aSelB plus outgroup datasets consisted of 311 and 298 aligned amino acid columns, respectively. Maximum likelihood (ML) phylogenetic analysis was carried out with RAxML v 7.0.4 [48] on the CIPRES portal v2.2 http://www.phylo.org/sub_sections/ portal/. The program was run with the PROTCATWAG model, with 100 bootstrap replicates. MrBayes v3.1.2 [49], also on the CIPRES portal v2.2, was run for 2 million generations, sampling every 100 generations, with 2 parallel runs of 8 chains each, using a gamma rate distribution with a mixed model that converged on WAG with a posterior probability of 0.99 for both data sets. A consensus tree was generated after discarding the first 200,000 generations from each run as a burn in. The standard deviation of split frequencies (SDSF) is shown in figure legends.

Genomic context was retrieved from the Entrez gene database [50], where records were available or via the CDS records within the NCBI genome entry. Protein structures were visualized and aligned with MacPyMOL.

Cysteine content was determined for archaeal predicted proteomes downloaded in Fasta format from the NCBI genome page http://www.ncbi.nlm.nih.gov/genomes/lproks.cgi. The percentage of cysteine in each proteome was calculated using a Python script.

\section{Additional material}

\begin{abstract}
Additional file 1: Baysian inference tree of aSelB and aSelBL with alF2 $\gamma$, bacterial SelB and EF-Tu as the outgroup. The tree was generated from 298 aligned amino acid positions. The SDSF at the end of the run was 0.02 Branch and tip labels are as per the Figure 1 legend. Additional file 2: Table showing distribution of aSelBL, aSelB, SeID and pylS across complete archaeal genomes. Square brackets indicate divergent or partial sequences (details in text). The percentage cysteine content of each proteome is indicated.

Additional file 3: Table of upstream and downstream genes from aSelBL and aSelB. Arrows ">" show the direction of gene relative $a S e I B L$ or aSelB. The " $X$ " at position 0 indicates the aSelBL or aSelB gene. HP stands for hypothetical protein, and is followed by additional details if it shares homology with a particular protein or domain. Genes with names in bold are transcribed in the same direction as aSelBL. Colours show homologous conserved flanking genes.

Additional file 4: Analysis of SelBL distribution corellation with cysteine content in archaeal proteomes. Colored circles represent data points for individual genomes (see Additional file 2), and the colour code for taxonomic groups is given in the inset box.
\end{abstract}

\section{Acknowledgements}

Many thanks to Andrey Konevega and Alena Paleskava for helpful discussion of the manuscript. This work was supported by Estonian Science Foundation grants (codes MJD99 to GCA, and 7616 to VH) and European Regional Development Fund through the Center of Excellence in Chemical Biology to $\mathrm{VH}$ and $\Pi \mathrm{T}$.

\section{Authors' contributions}

GCA conceived of the study, carried out sequence searching and phylogenetic analyses, and drafted the manuscript. VH participated in drafting the manuscript and in the analysis of cysteine content distribution. $T T$ helped draft the manuscript and participated in the coordination of the study. All authors read and approved the final manuscript.

Received: 8 November 2010 Accepted: 21 January 2011

Published: 21 January 2011

\section{References}

1. Yatime L, Schmitt E, Blanquet S, Mechulam Y: Functional molecular mapping of archaeal translation initiation factor 2. J Biol Chem 2004, 279:15984-15993. 
2. Ohtsuki T, Watanabe Y: T-armless tRNAs and elongated elongation factor Tu. IUBMB Life 2007, 59:68-75.

3. Ohtsuki T, Sato A, Watanabe $Y$, Watanabe $K$ : A unique serine-specific elongation factor Tu found in nematode mitochondria. Nat Struct Biol 2002, 9:669-673.

4. Keeling P, Fast N, McFadden G: Evolutionary relationship between translation initiation factor elF-2gamma and selenocysteine-specific elongation factor SELB: change of function in translation factors. $J \mathrm{Mol}$ Evol 1998, 47:649-655.

5. Paleskava A, Konevega AL, Rodnina MV: Thermodynamic and kinetic framework of selenocysteyl-tRNASec recognition by elongation factor SelB. J Biol Chem 2009, 285:3014-3020.

6. Nauser T, Steinmann D, Koppenol WH: Why do proteins use selenocysteine instead of cysteine? Amino acids.

7. Kromayer M, Wilting R, Tormay P, Bock A: Domain structure of the prokaryotic selenocysteine-specific elongation factor SelB. J Mol Biol 1996, 262:413-420.

8. Copeland PR, Fletcher JE, Carlson BA, Hatfield DL, Driscoll DM: A novel RNA binding protein, SBP2, is required for the translation of mammalian selenoprotein mRNAs. EMBO J 2000, 19:306-314.

9. Donovan J, Copeland PR: Evolutionary history of selenocysteine incorporation from the perspective of SECIS binding proteins. BMC Evol Biol 2009, 9:229.

10. Romero H, Zhang Y, Gladyshev VN, Salinas G: Evolution of selenium utilization traits. Genome Biol 2005, 6:R66.

11. Leipe D, Wolf $Y$, Koonin E, Aravind L: Classification and evolution of P-loop GTPases and related ATPases. J Mol Biol 2002, 317:41-72.

12. Atkins JF, Gesteland R: Biochemistry. The 22nd amino acid. Science 2002, 296:1409-1410.

13. Srinivasan G, James CM, Krzycki JA: Pyrrolysine encoded by UAG in Archaea: charging of a UAG-decoding specialized tRNA. Science 2002, 296:1459-1462.

14. Ibba M, Soll D: Aminoacyl-tRNAs: setting the limits of the genetic code. Genes Dev 2004, 18:731-738.

15. Crystal Structure Of The Selb-Like Elongation Factor Ef-Pyl From Methanosarcina Mazei. [http://www.pdb.org/pdb/explore/explore.do? structureld=2ELF].

16. Theobald-Dietrich A, Frugier M, Giege R, Rudinger-Thirion J: Atypical archaeal tRNA pyrrolysine transcript behaves towards EF-Tu as a typical elongator tRNA. Nucleic Acids Res 2004, 32:1091-1096.

17. Brochier-Armanet C, Boussau B, Gribaldo S, Forterre P: Mesophilic crenarchaeota: proposal for a third archaeal phylum, the Thaumarchaeota. Nature reviews Microbiology 2008, 6:245-252.

18. Baker BJ, Comolli LR, Dick GJ, Hauser LJ, Hyatt D, Dill BD, Land ML, Verberkmoes NC, Hettich RL, Banfield JF: Enigmatic, ultrasmall, uncultivated Archaea. Proc Natl Acad Sci USA 2010, 107:8806-8811.

19. Gribaldo S, Brochier C: Phylogeny of prokaryotes: does it exist and why should we care? Research in Microbiology 2009, 160:513-521.

20. Yuan J, O'Donoghue P, Ambrogelly A, Gundllapalli S, Sherrer RL, Palioura S, Simonovic M, Soll D: Distinct genetic code expansion strategies for selenocysteine and pyrrolysine are reflected in different aminoacyl-tRNA formation systems. FEBS Lett 2010, 584:342-349.

21. Haft DH, Self WT: Orphan SelD proteins and selenium-dependent molybdenum hydroxylases. Biol Direct 2008, 3:4.

22. Bourne H, Sanders D, McCormick F: The GTPase superfamily: conserved structure and molecular mechanism. Nature 1991, 349:117-127.

23. Sprang SR: G protein mechanisms: insights from structural analysis. Annu Rev Biochem 1997, 66:639-678.

24. Voorhees RM, Schmeing TM, Kelley AC, Ramakrishnan V: The mechanism for activation of GTP hydrolysis on the ribosome. Science 2010, 330:835-838.

25. Nissen P, Kjeldgaard M, Thirup S, Polekhina G, Reshetnikova L, Clark BF, Nyborg J: Crystal structure of the ternary complex of Phe-tRNAPhe, EFTu, and a GTP analog. Science 1995, 270:1464-1472.

26. Nissen $P$, Thirup $S$, Kjeldgaard $M$, Nyborg J: The crystal structure of CystRNACys-EF-Tu-GDPNP reveals general and specific features in the ternary complex and in tRNA. Structure 1999, 7:143-156.

27. Leibundgut M, Frick C, Thanbichler M, Böck A, Ban N: Selenocysteine tRNAspecific elongation factor SelB is a structural chimaera of elongation and initiation factors. EMBO J 2005, 24:11-22.
28. Schmeing TM, Voorhees RM, Kelley AC, Gao YG, Murphy FVt, Weir JR, Ramakrishnan V: The crystal structure of the ribosome bound to EF-Tu and aminoacyl-tRNA. Science 2009, 326:688-694.

29. Price M, Arkin A, Alm E: The life-cycle of operons. PLoS Genet 2006, 2:e96.

30. Zhang Y, Baranov PV, Atkins JF, Gladyshev VN: Pyrrolysine and selenocysteine use dissimilar decoding strategies. J Biol Chem 2005, 280:20740-20751.

31. Garrett RA, Dalgaard J, Larsen N, Kjems J, Mankin AS: Archaeal rRNA operons. Trends Biochem Sci 1991, 16:22-26.

32. Lechner K, Heller G, Bock A: Organization and nucleotide sequence of a transcriptional unit of Methanococcus vannielii comprising genes for protein synthesis elongation factors and ribosomal proteins. J Mol Evol 1989, 29:20-27.

33. Stock T, Rother M: Selenoproteins in Archaea and Gram-positive bacteria. Biochim Biophys Acta 2009, 1790:1520-1532.

34. Yuan J, Hohn MJ, Sherrer RL, Palioura S, Su D, Soll D: A tRNA-dependent cysteine biosynthesis enzyme recognizes the selenocysteine-specific tRNA in Escherichia coli. FEBS Lett 2010, 584:2857-2861.

35. Major TA, Burd H, Whitman WB: Abundance of $4 \mathrm{Fe}-4 \mathrm{~S}$ motifs in the genomes of methanogens and other prokaryotes. FEMS Microbiol Lett 2004, 239:117-123

36. Sauerwald A, Zhu W, Major TA, Roy H, Palioura S, Jahn D, Whitman WB, Yates JR, Ibba M, Soll D: RNA-dependent cysteine biosynthesis in archaea. Science 2005, 307:1969-1972.

37. O'Donoghue P, Sethi A, Woese CR, Luthey-Schulten ZA: The evolutionary history of Cys-tRNACys formation. Proc Natl Acad Sci USA 2005, 102:19003-19008.

38. Qu G, Wang W, Chen LL, Qian SS, Zhang HY: tRNA-dependent cysteine biosynthetic pathway represents a strategy to increase cysteine contents by preventing it from thermal degradation: thermal adaptation of methanogenic archaea ancestor. J Biomol Struct Dyn 2009, 27:111-114.

39. Klipcan L, Frenkel-Morgenstern M, Safro MG: Presence of tRNA-dependent pathways correlates with high cysteine content in methanogenic Archaea. Trends Genet 2008, 24:59-63.

40. Dell VA, Miller DL, Johnson AE: Effects of nucleotide- and aurodoxinduced changes in elongation factor Tu conformation upon its interactions with aminoacyl transfer RNA. A fluorescence study. Biochemistry 1990, 29:1757-1763.

41. Kapp LD, Lorsch JR: GTP-dependent recognition of the methionine moiety on initiator tRNA by translation factor elF2. J Mol Biol 2004, 335:923-936

42. Gromadski KB, Schummer T, Stromgaard A, Knudsen CR, Kinzy TG, Rodnina MV: Kinetics of the interactions between yeast elongation factors $1 \mathrm{~A}$ and $1 \mathrm{Balpha}$, guanine nucleotides, and aminoacyl-tRNA. J Biol Chem 2007, 282:35629-35637.

43. Leinfelder W, Zehelein E, Mandrand-Berthelot MA, Bock A: Gene for a novel tRNA species that accepts L-serine and cotranslationally inserts selenocysteine. Nature 1988, 331:723-725.

44. Dmitriev SE, Terenin IM, Andreev DE, Ivanov PA, Dunaevsky JE, Merrick WC, Shatsky IN: GTP-independent tRNA delivery to the ribosomal P-site by a novel eukaryotic translation factor. J Biol Chem 2010, 285:26779-26787.

45. Heinemann IU, Soll D, Randau L: Transfer RNA processing in archaea: Unusual pathways and enzymes. FEBS letters 2010, 584:303-309.

46. Atkinson G, Baldauf S: Evolution of the Translational GTPase Superfamily. J Biomolecular Structure \& Dynamics 2009, 26:841-842.

47. Katoh $\mathrm{K}$, Kuma $\mathrm{K}$, Toh H, Miyata T: MAFFT version 5: improvement in accuracy of multiple sequence alignment. Nucleic Acids Res 2005, 33:511-518.

48. Stamatakis A: RAxML-VI-HPC: maximum likelihood-based phylogenetic analyses with thousands of taxa and mixed models. Bioinformatics 2006, 22:2688-2690.

49. Huelsenbeck JP, Ronquist F: MRBAYES: Bayesian inference of phylogenetic trees. Bioinformatics 2001, 17:754-755.

50. Maglott D, Ostell J, Pruitt K, Tatusova T: Entrez Gene: gene-centered information at NCBI. Nucleic Acids Res 2007, 35:D26-31.

doi:10.1186/1471-2148-11-22

Cite this article as: Atkinson et al: An ancient family of SelB elongation factor-like proteins with a broad but disjunct distribution across archaea. BMC Evolutionary Biology 2011 11:22 